\title{
Bremsstrahlung from relativistic heavy ions in matter
}

\author{
Allan H. Sørensen \\ Department of Physics and Astronomy, University of Aarhus, DK-8000 Aarhus C, Denmark
}

(Received 24 September 2009; published 8 February 2010)

\begin{abstract}
The emission of electromagnetic radiation by relativistic bare heavy ions penetrating ordinary matter is investigated. Our main aim is to determine the bremsstrahlung which we define as the radiation emitted when the projectile does not break up. It pertains to collisions without nuclear contact ("ultraperipheral collisions"). Requirement of coherent action of the nucleons in order to keep the penetrating projectile intact limits bremsstrahlung to relatively soft photons. The spectrum shows a resonance structure with peak position near $2 \gamma$ times the position of the giant dipole resonance, that is, near $25 \gamma \mathrm{MeV}$ for a lead ion $\left(\gamma \equiv E / M c^{2}\right.$ is the Lorentz factor of the projectile of energy $E$ and mass $M$ ). The maximum exceeds the bremsstrahlung from a hypothetical structureless, pointlike particle of the same charge and mass as the incoming nucleus, but rapid depletion follows on the high-energy side of the peak. As a result of its relative softness, bremsstrahlung never dominates the energy-loss process for heavy ions. As to the emission of electromagnetic radiation in collisions with nuclear break-up, it appears modest when pertaining to incoherent action of the projectile nucleons in noncontact collisions. In collisions with nuclear contact, though, substantial radiation is emitted. It overshoots the bremsstrahlung. However, despite the violence of contact events, the associated photon emission only exceeds the radiation from a hypothetical structureless pointlike nucleus [emitted energy per unit photon-energy interval essentially constant up to $(\gamma-1) M c^{2}$ ] at relatively low photon energies (for lead roughly below $0.2 \gamma \mathrm{GeV}$, a limit which is about an order of magnitude above the position of the bremsstrahlung peak). Results are presented for bare lead ions penetrating a solid lead target at energies of $158 \mathrm{GeV} / \mathrm{n}(\gamma=170)$ and beyond.
\end{abstract}

DOI: 10.1103/PhysRevA.81.022901 PACS number(s): 34.50.Bw, 41.60.-m, 25.75.-q, 78.70.-g

\section{INTRODUCTION}

Emission of bremsstrahlung is the primary cause of slowing down for relativistic electrons penetrating a substance at high energies, that is, at energies beyond, roughly, $800 \mathrm{MeV} /\left(Z_{t}+\right.$ 1.2), where $Z_{t}$ is the atomic number of the target. Theoretical studies of energy-loss processes for relativistic bare heavy ions have pointed toward a similar dominance of radiative losses at energies far beyond the rest energy of the heavy projectile $[1,2]$. However, these studies turn out to overestimate the radiative losses substantially by neglecting the composite nature of the projectile $[3,4]$. The same holds true for the bremsstrahlung intensities calculated in Ref. [5]. A rough estimate presented in Ref. [4] shows that as a result of strong moderation due to internal structure, bremsstrahlung never dominates the energy-loss process for a bare nucleus: For bare relativistic heavy ions, atomic excitation and ionization are the most important energy-loss processes up to a relatively high energy beyond which electron-positron pair creation becomes the major energy-loss channel.

The emission of bremsstrahlung reflects fundamental aspects of the nature of the emitter as well as of its electromagnetic interaction with the surroundings. Hence, in this article, we shall go beyond the simple estimate given in Ref. [4] and attempt to assess fairly accurately the bremsstrahlung spectrum for a bare heavy ion penetrating matter. The intention is to predict the true spectrum within $\pm 10 \%$.

When an energetic atomic nucleus penetrates matter, it may or may not break up in the interaction with a given target constituent. Electromagnetic radiation is generally emitted in both cases. In this article, we shall only put the label bremsstrahlung on the radiation in case the projectile remains intact throughout the interaction. This reflects the very meaning of the word: radiation causing moderation of the motion of the projectile (braking). Our use is opposed to the less restrictive use prevailing in a major part of the literature where the label bremsstrahlung is applied also to cases where the projectile decomposes completely.

With our terminology, bremsstrahlung pertains to collisions without contact between projectile and target nuclei, so-called ultraperipheral collisions. In collisions with nuclear interaction, the collision partners break up and violent acceleration of the charged nuclear constituents leads to substantial radiation. To complete the picture of electromagnetic radiation by a relativistic atomic nucleus penetrating matter, we shall estimate the emission in such events as well as hint at additional photon sources for noncontact collisions.

\section{REFERENCE CROSS SECTION}

As a reference for the results following in later sections, let us quote a simple formula for the bremsstrahlung intensity for a pointlike and structureless particle impinging on a fixed scattering center at relativistic energy. The formula is obtained in a classical calculation with photon energy and momentum included in the energy and momentum balance [6]. For a projectile of mass $M=A M_{u}$ ( $A$ is mass number and $M_{u}=931.5 \mathrm{MeV} / c^{2}$ the atomic mass unit), charge $Z e$, energy $E \gg M c^{2}$, and a center of charge $Z_{t} e$, the so-called radiation cross section amounts to

$$
\frac{d \chi}{d \hbar \omega}=\frac{16}{3} \frac{Z_{t}^{2} Z^{4}}{A^{2}} \alpha r_{u}^{2} L,
$$

where $\alpha \equiv e^{2} / \hbar c$ is the fine-structure constant and $r_{u} \equiv$ $e^{2} / M_{u} c^{2}$ the classical nucleon radius. Note that the differential radiation cross section (notation adapted from Ref. [6]) has dimensions of (energy $\times$ area/energy $=$ area), the cross section for photon emission per unit energy interval is obtained 
by dividing with the photon energy $\hbar \omega$. In Eq. (1), the factor $L$ is essentially the logarithm of the ratio between the effective maximum and minimum momentum transfers to the scattering center. For a bare pointlike Coulomb center, it is given as

$$
L \simeq \ln \left[\frac{E(E-\hbar \omega)}{M c^{2} \hbar \omega}\right] .
$$

For a pointlike Coulomb center screened at the Thomas-Fermi distance corresponding to atomic number $Z_{t}$, the logarithmic factor modifies to

$$
\begin{gathered}
L \simeq \ln \left(\frac{233 M}{Z_{t}^{1 / 3} m}\right)-\frac{1}{2}\left[\ln \left(1+r^{2}\right)-\frac{1}{1+r^{-2}}\right], \\
r=\frac{96 \hbar \omega}{\gamma \gamma^{\prime} Z_{t}^{1 / 3} m c^{2}},
\end{gathered}
$$

where $\gamma \equiv E / M c^{2}$ and $\gamma^{\prime} \equiv(E-\hbar \omega) / M c^{2}$ are the Lorentz factors of the projectile before and after emission and $m$ is the electron mass. The quantity $r$ is the ratio between the effective minimum momentum transfers calculated without and with screening included. When $r \ll 1$, the logarithmic factor $L$ is given by the first term in (3). For $Z_{t}=Z=82, A=208$, and $r=0$, the radiation cross section (1) amounts to 0.110 barn. The limit where $r$ is small for essentially all frequencies is known as the limit of complete screening. For $\gamma=\gamma^{\prime}=170$ and $Z=82$, the ratio $r$ assumes the value $r=\hbar \omega / 0.67 \mathrm{GeV}$.

The radiation cross section (1) with $L$ given by (3) will be used as a common reference value. We refer the reader to Heitler's book [7] for the bremsstrahlung spectrum resulting from a full quantal calculation. For soft photons $\hbar \omega \ll E$ changes are limited to modest changes in the argument of the logarithm (3). For finite values of $\hbar \omega / E$, an additional factor of $\left[1-\hbar \omega / E+3(\hbar \omega / E)^{2} / 4\right]$ appears in the radiation cross section (1). Note that except for the modest variation of the latter factor and the logarithm, the spectrum is flat all way up to the primary energy.

In the limit of complete screening, the argument of the logarithm (3) is, up to a factor of $\simeq 2$, simply the ratio of the screening distance to the Compton wavelength $\lambda_{C} \equiv \hbar / M c$ of the projectile. This reveals that bremsstrahlung for the pointlike projectile is composed of contributions from impact parameters which effectively range between these limits. If the screening is not complete, the upper limit is modified (that is, it is less than the Thomas-Fermi length of the target atom), but the lower limit remains the same. However, due to the large mass, the Compton wavelength of a heavy nucleus is only a small fraction of the nuclear radius. With the latter given as

$$
R=1.18 \mathrm{fm} \times A^{1 / 3},
$$

the ratio is $\lambda_{C} / R \simeq 0.2 / A^{4 / 3}$ which obviously is very small for high values of $A$. The requirement of no nuclear contact implies replacement of the Compton wavelength by the sum of the radii of the colliding nuclei as the effective minimum impact parameter. This leads to a lower value of the bremsstrahlung logarithm $L$. The composite nature of the projectile leads to additional very significant changes in the spectrum, as we shall see.

In his review on energy loss of relativistic heavily ionizing particles, Ahlen [1] simply cites the result (1) for a rough comparison with other loss channels (applying only the first term in the logarithm (3) pertaining to the limit of complete screening). As a result, his estimates are far too high (and actually inconsistent since the complete screening limit is far from being reached in the cases considered in his table XI).

Weaver and Westphal [2] apply Heitler's quantal result for electrons (impinging on a bare Coulomb center) with replacement of the electron charge and mass by those of the projectile nucleus. As a consequence, the authors conclude that bremsstrahlung can become important in energy loss for very highly charged ions at energies which are accessible to present-generation heavy-ion accelerators. As we shall see, this does not hold true.

Bertulani and Baur [5] perform a calculation based on classical electrodynamics of the bremsstrahlung emitted in the collision between two bare heavy nuclei. They include the restriction that the impact parameter be in excess of the sum of the nuclear radii, but otherwise the collision partners are treated as pointlike structureless objects. As a result, their radiation cross section comes out as a virtual photon intensity times the classical Thomson cross section for a pointlike particle with mass and charge identical to those of either nucleus. As will be clear from the following discussion, this underestimates the emission of relatively soft photons but overestimates hard-photon emission. If the radiative energy loss were evaluated, it would come out much higher than our results.

\section{NONCONTACT COLLISIONS}

To determine the bremsstrahlung emitted in ultraperipheral collisions, we shall apply the Weizsäcker-Williams (WW) method of virtual quanta [6]. In general there are two contributions to the emission, one from the scattering of the virtual photons of the projectile on the target, and one from the scattering of the virtual photons of the target on the projectile. The latter process is considered in the rest frame of the incoming particle with the scattered photons subsequently transformed to the laboratory. As is apparent from Heitler's discussion of bremsstrahlung in electron-electron collisions [7], scattering on the projectile brings the major contribution. When we aim for stopping, we require the projectile to stay intact; processes where the projectile breaks up will be considered as separate events. This translates into a requirement of coherent action of the constituents; if the recoil in scattering were to be taken up by a single proton, this proton would in general leave the nucleus.

\section{A. Elastic photon scattering}

There are five characteristic energies in the scattering of WW photons on the projectile, three in the $\mathrm{MeV}$ range and two of much higher values. Among the latter two, one pertains to the transition from classical to quantal scattering, and the other to the cut-off of the WW spectrum. As we shall see shortly, only the scattering of much softer photons brings substantial contributions to the bremsstrahlung cross section which therefore may be determined classically on the basis of the low-energy expansion of the WW spectrum. Among the remaining three characteristic energies are $\hbar \omega_{1}$ which 
distinguishes scattering on a single rigid object of charge $Z e$ and mass $M\left(\omega<\omega_{1}\right)$ from coherent scattering on $Z$ quasi-free protons each of mass $M_{p}\left(\omega>\omega_{1}\right)$, and $\hbar \omega_{2}$ beyond which incoherent scattering on individual quasi-free protons is possible. A typical value for $\hbar \omega_{1}$ is $\sim 8 \mathrm{MeV}$ (binding energies per nucleon are of this order). The energy $\hbar \omega_{2}$ is defined by the wavelength being comparable to the nuclear size $R$ of the projectile, that is, $\hbar \omega_{2} \simeq \hbar c / R$ which amounts to $25-30 \mathrm{MeV}$ for the heaviest nuclei.

To obtain the photon scattering cross section, we shall as a first attempt assume $\omega_{1}$ and $\omega_{2}$ to divide sharply between the different physical situations just described. In this approximation, the scattering cross section in the projectile rest frame $\mathcal{R}$ (primed variables) is

$$
\frac{d \sigma}{d \Omega^{\prime}}=\left\{\begin{array}{cl}
\left(\frac{(Z e)^{2}}{M c^{2}}\right)^{2} \frac{1}{2}\left(1+\cos ^{2} \psi^{\prime}\right) ; & \omega^{\prime}<\omega_{1} \\
Z^{2}\left(\frac{e^{2}}{M_{p} c^{2}}\right)^{2} \frac{1}{2}\left(1+\cos ^{2} \psi^{\prime}\right) ; & \omega_{1}<\omega^{\prime}<\omega_{2} \\
Z^{2}\left(\frac{e^{2}}{M_{p} c^{2}}\right)^{2} \frac{1}{2}\left(1+\cos ^{2} \psi^{\prime}\right) \Theta\left(1-2 \frac{\omega^{\prime}}{\omega_{2}} \sin \frac{\psi^{\prime}}{2}\right) ; & \omega_{2}<\omega^{\prime}
\end{array}\right\}
$$

or, more conveniently,

$$
\frac{d \sigma}{d \Omega^{\prime}}=Z^{2} r_{p}^{2} \frac{1}{2}\left(1+\cos ^{2} \psi^{\prime}\right) \times\left\{\begin{array}{cl}
\left(\frac{Z M_{p}}{M}\right)^{2} ; & \omega^{\prime}<\omega_{1} \\
1 ; & \omega_{1}<\omega^{\prime}<\omega_{2} \\
\Theta\left(1-2 \frac{\omega^{\prime}}{\omega_{2}} \sin \frac{\psi^{\prime}}{2}\right) ; & \omega_{2}<\omega^{\prime}
\end{array}\right\}
$$

where $r_{p} \equiv e^{2} / M_{p} c^{2}$ is the classical radius of the proton. See Ref. [6] for the individual components. Since the virtual photons of the target are aimed against the projectile velocity $\mathbf{v}$, the scattering angle $\psi^{\prime}$ is measured relative to $-\mathbf{v}$, whereby the photon-emission angle relative to $\mathbf{v}$ is $\theta^{\prime}=\pi-\psi^{\prime}$. The last factor for the high-energy range (the Heaviside or step function $\Theta ; \Theta=1$ for positive arguments while $\Theta=0$ for negative) restricts scattering to angles sufficiently small that the change in wave number times the size of the nucleus is small compared to 1 , that is, to angles $\psi^{\prime}$ smaller than approximately $\omega_{2} / \omega^{\prime}$. This is the requirement for coherence; see Ref. [6]. The restriction by the Heaviside function implies that, after integration over scattering angles, the cross section falls off beyond $\omega_{2}$. Since $\hbar \omega_{2}$ is much smaller than the mass of all scatterers involved, the lightest being a proton, quantum effects may be neglected and the classical expression for scattering (6) suffices. The estimates of the energy loss through emission of bremsstrahlung presented in Ref. [4] were based on the approximation (6).

Application of a sharp divide at $\omega^{\prime}=\omega_{1}$ implies a discontinuity of the scattering cross section at this frequency. For heavy nuclei, the charge and mass-dependent factor for the low-frequency range in Eq. (6) is about one fourth to one sixth of the factor pertaining to scattering at higher frequencies $\left(Z / M \simeq Z / A M_{p} \simeq 1 / 2 M_{p}\right)$; that is, coherent scattering on $Z$ quasi-free protons is about four to six times as effective as scattering on a pointlike nucleus of charge $Z e$. This difference is related to the fact that in the case of the pointlike nucleus, the incoming wave has to accelerate the entire nuclear mass, whereas for scattering on $Z$ quasi-free protons, the neutrons are left inactivated.

Besides being discontinuous at $\omega^{\prime}=\omega_{1}$, the cross section (6) lacks one important feature: a resonance. To model photon scattering by the protons of the projectile nucleus as photon scattering by a collection of $Z$ effectively free charged particles is correct only asymptotically at high photon energies $\omega^{\prime} \gg$ $\omega_{1}$. In the transition between the asymptotes, that is, from scattering on a rigid pointlike nucleus with all constituents tightly bound, to scattering on the collection of $Z$ free protons, a resonance will show up. The characteristic energy of this resonance is the final characteristic energy for elastic photon scattering. It will roughly be the energy of the peak of the giant dipole resonance (GDR) in photoabsorption. This places it in the interval between $\omega_{1}$ and $\omega_{2}$.

For ${ }^{208} \mathrm{~Pb}$ projectiles, we have obtained a useful and quite accurate cross section based on the experimental data for elastic photon scattering given by Schelhaas et al. [8]. These data cover the energy range $10-100 \mathrm{MeV}$, and results for four scattering angles are presented. Near $\hbar \omega_{2}=28.2 \mathrm{MeV}$ recordings are comparable to Eq. (6) at all angles; for $\omega_{1}<$ $\omega^{\prime}<\omega_{2}$, the data overshoot the simple asymptotic expression by up to nearly a factor of 10 , and for $\omega^{\prime}>\omega_{2}$, the data show a fall-off as does the simple expression. The resonance may be represented quite well by that pertaining to classical scattering by an oscillator (see, e.g., Heitler [7] for the latter). The depletion at high energies may be represented by a simple exponential behavior which actually may be extended to energies somewhat below $\hbar \omega_{2}$. Our model cross section for ${ }^{208} \mathrm{~Pb}$ is

$$
\frac{d \sigma}{d \Omega^{\prime}}=Z^{2} r_{p}^{2} \frac{1}{2}\left(1+\cos ^{2} \psi^{\prime}\right) \times\left\{\begin{array}{cl}
\left(\frac{Z M_{p}}{M}\right)^{2} ; & \hbar \omega^{\prime}<\hbar \omega_{1} \\
0.793 \frac{\left(\hbar \omega^{\prime}\right)^{4}}{\left.\left(\hbar \omega^{\prime}\right)^{2}-\left(E_{m}\right)^{2}\right)^{2}+\left(\Gamma \hbar \omega^{\prime}\right)^{2}} ; & \hbar \omega_{1}<\hbar \omega^{\prime}<\hbar \tilde{\omega}_{2} \\
1.93 \exp \left(-\epsilon\left(\hbar \omega^{\prime}-\hbar \tilde{\omega}_{2}\right) \sin ^{2} \frac{\psi^{\prime}}{2}\right) ; & \hbar \tilde{\omega}_{2}<\hbar \omega^{\prime}
\end{array}\right\},
$$


where $Z=82$, that is, $Z^{2} r_{p}^{2}=0.1584$ mbarn, and $M=$ $208 M_{u}$. The parameters for the resonance are $E_{m}=13.7 \mathrm{MeV}$ and $\Gamma=4.15 \mathrm{MeV}$, and the value of the high-energy depletion factor is $\epsilon=0.11 \mathrm{MeV}^{-1}$. With the resonance part fixed (front factor as well as the values of $E_{m}$ and $\Gamma$ ), the cross section is continuous at $\hbar \omega_{1}$ with the choice $7.69 \mathrm{MeV}$ for this energy. Similarly, with the other dividing energy chosen as $\hbar \tilde{\omega}_{2}=$ 22.0 MeV, the cross section is continuous here as well with the choice of front factor displayed in the last line of Eq. (7). Generally, the fit (7) reproduces the experimental data within $\sim 10 \%$. Exceptions are the rapid increase at the low-energy side of the resonance (where the fit generally falls below the data during the first half of the rise) and the depletion at high energies for the lowest angle at which data are collected (where the fit falls off somewhat too slowly). However, the differences do not play a major role for the radiation cross section to be derived below since transformation to the laboratory effectively implies integration over a range of energies and angles whereby average errors rather than individual errors count.

\section{B. Virtual photon intensity}

To get the emitted energy per frequency bin in the laboratory, the scattering cross section (6) or (7) is multiplied by the virtual photon intensity spectrum in $\mathcal{R}$, and the product is transformed to the laboratory and integrated over angles. Neglecting contributions of order $1 / \gamma^{2}$, the WW spectrum is given as [6]

$$
\frac{d I^{\prime}}{d \hbar \omega^{\prime}}=\frac{2}{\pi} \alpha Z_{t}^{2}\left\{\xi^{\prime} K_{0}\left(\xi^{\prime}\right) K_{1}\left(\xi^{\prime}\right)+\frac{1}{2} \xi^{\prime 2}\left[K_{0}^{2}\left(\xi^{\prime}\right)-K_{1}^{2}\left(\xi^{\prime}\right)\right]\right\},
$$

where $\xi^{\prime}$ is defined as

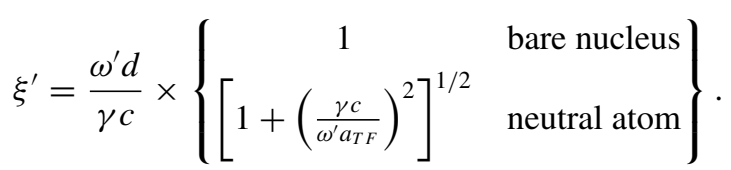

The result for a neutral target atom is derived for an exponentially screened Coulomb potential with the Thomas-Fermi length of the target $a_{T F}$ chosen as the screening length; see the appendix. Of course the true screening is not exponential, but since the screening length basically ends up in the argument of a fairly large logarithm [see (10) below], details of the screening are not important (as long as we do not aim for reducing errors below a few percent). The transition between the expressions pertaining to a fully screened and a bare target nucleus with increasing photon energy happens for $\hbar \omega^{\prime} \sim \gamma \hbar c / a_{T F}$ which varies between $4.2 \times 10^{-3} \gamma \mathrm{MeV}$ and $2.0 \times 10^{-2} \gamma \mathrm{MeV}$ according to the value of $Z_{t}$. For the heaviest targets and $\gamma=170$, the value is $3.3 \mathrm{MeV}$ or about one third of $\hbar \omega_{1}$, implying screening is of little importance. For $\gamma=3000$, the value is slightly above $\hbar \omega_{2}$ for the heaviest targets implying influence of atomic screening on the bremsstrahlung spectrum. In noncontact collisions, the minimum impact parameter $d$ is defined by the sum of the radii of the colliding nuclei, $d \equiv R_{\Sigma}$. Since the modified Bessel functions $K_{0}$ and $K_{1}$ fall off exponentially for arguments larger than 1, the effective maximum photon energy in the WW spectrum is $\gamma \hbar c / R_{\Sigma}$ which, for comparable sizes of the colliding nuclei, amounts to roughly $\gamma \hbar \omega_{2} / 2$. The scattering cross section falls off for $\omega^{\prime}>\omega_{2}$, and hence for high values of $\gamma$, it is depleted long before the photon spectrum is cut off. As a result, $\xi^{\prime}$ is effectively small where the cross section is of significance and the WW spectrum may be represented by its low-energy asymptote obtained by application of the small-argument expansions of the modified Bessel functions,

$$
\frac{d I^{\prime}}{d \hbar \omega^{\prime}}=\frac{2}{\pi} \alpha Z_{t}^{2}\left\{\ln \frac{1.123}{\xi^{\prime}}-\frac{1}{2}\right\} \equiv \frac{2}{\pi} \alpha Z_{t}^{2} \ln \frac{C}{\xi^{\prime}} .
$$

The last transcription is introduced for later convenience, $C \equiv$ $2 / \exp \left(\gamma_{E}+\frac{1}{2}\right)=0.681 \ldots$, where $\gamma_{E}=0.5772 \ldots$ is Euler's constant.

The application of a sharp cut in impact parameter $b$ at $R_{\Sigma}=R+R_{t}$ is an approximation. Lack of nuclear contact does not imply that the projectile necessarily survives a collision. In particular, it may break up through electromagnetic dissociation (see Refs. [9,10] and references therein). Immediately outside $R_{\Sigma}$, this process is so strong that it may effectively reduce the number of surviving projectile ions. We will consider ${ }^{208} \mathrm{~Pb}$ ions penetrating a lead target. For LHC energies $(\gamma=3000)$, a computation along the lines laid out in Ref. [9] gives at $b=R_{\Sigma}$ a probability of $36 \%$ for absorbing a single photon with energy above the neutron-separation energy and $68 \%$ for absorbing one or more such photons, leaving only $32 \%$ of the collisions without any such photons absorbed. To increase the fraction of collisions without photon absorption to $90 \%$ requires an impact parameter of $3.2 R_{\Sigma}, 99 \%$ similarly requires $b=10.0 R_{\Sigma}$. For $\gamma=170$, the fraction with no photons absorbed above the neutron-separation energy is $38 \%$ at $b=R_{\Sigma}, 90 \%$ at $b=2.9 R_{\Sigma}$, and $99 \%$ at $b=8.6 R_{\Sigma}$. To account approximately for the depletion due to electromagnetic dissociation, the virtual photon intensity computed at a given impact parameter $d I / d \omega d^{2} b$ (cf. the appendix) could be multiplied by the probability $P^{(0)}(b)$ for no photons absorbed before the integration over impact parameters beyond the minimum $d \equiv R_{\Sigma}$ is performed. Such a procedure reduces the results presented in Figs. 2 and 3 by $7-8 \%$. That is, despite large probabilities for dissociation immediately outside $R_{\Sigma}$, we may expect to hit true intensities closer than $10 \%$ by maintaining a sharp cut at $R_{\Sigma}$ and apply Eqs. (8)-(10) with this value for $d$.

\section{Transformation to lab}

In the rest frame $\mathcal{R}$ of the projectile, the product of the virtual photon intensity of the target object and the photon scattering cross section produces the double-differential radiation cross section,

$$
\frac{d^{2} \chi^{\prime}}{d \hbar \omega^{\prime} d \Omega^{\prime}}=\frac{d \sigma}{d \Omega^{\prime}} \frac{d I^{\prime}}{d \hbar \omega^{\prime}}
$$

Transformation from $\mathcal{R}$ to the laboratory may be performed by means of the result [6]

$$
\frac{1}{\omega^{2}} \frac{d^{2} \chi}{d \hbar \omega d \Omega}=\frac{1}{\omega^{\prime 2}} \frac{d^{2} \chi^{\prime}}{d \hbar \omega^{\prime} d \Omega^{\prime}} .
$$

From the relativistic Doppler shift formula $\omega^{\prime}=\gamma \omega(1-$ $\beta \cos \theta$ ), where $\theta$ denotes the angle of photon emission relative 
to the direction of motion of the projectile, we have

$$
\frac{\omega}{\omega^{\prime}}=\frac{1}{\gamma(1-\beta \cos \theta)} \text {. }
$$

The inverse Doppler formula combined with (13) gives

$$
\cos \theta^{\prime}=\frac{\cos \theta-\beta}{1-\beta \cos \theta}
$$

by use of $1 / \gamma^{2}=1-\beta^{2}$, where $\beta \equiv v / c$.

Generally, emission angles in the laboratory are rather small for high values of $\gamma$ : As is evident from the inverse of (14) scattering at right angles in $\mathcal{R}$ corresponds to $\cos \theta=\beta$, that is, to

$$
\sin ^{2} \theta=1 / \gamma^{2} \ll 1,
$$

and since the scattering cross section in $\mathcal{R}$ is approximately symmetric around $\pi / 2$ (at least for $\omega^{\prime}<\omega_{2}$ ), typical emission angles in the laboratory are $\gamma \theta \sim 1$. For small angles, the transformations (13)-(14) reduce to

$$
\frac{\omega}{\omega^{\prime}} \simeq \frac{2 \gamma}{1+\gamma^{2} \theta^{2}}, \quad \cos \theta^{\prime} \simeq \frac{1-\gamma^{2} \theta^{2}}{1+\gamma^{2} \theta^{2}} .
$$

From these relations, we may deduce a couple of further results to be used in the transformation to the laboratory:

$$
\left(\frac{\omega}{\omega^{\prime}}\right)^{2} \frac{1}{2}\left(1+\cos ^{2} \psi^{\prime}\right) \simeq 4 \gamma^{2} \frac{1+\gamma^{4} \theta^{4}}{\left(1+\gamma^{2} \theta^{2}\right)^{4}},
$$

$$
\Xi=\left\{\begin{array}{c}
\left(\frac{Z M_{p}}{M}\right)^{2} ; \\
1 ; \\
\Theta\left[\frac{1}{4}\left(\frac{2 \gamma \omega_{2}}{\omega}\right)^{2}-\left(1+\gamma^{2} \theta^{2}\right)\right]
\end{array}\right.
$$

For any fixed photon frequency in the laboratory, each of the three conditions listed in Eq. (21) defines an allowed range of emission angles $\theta$, if any. Integration of Eq. (19) over these angles gives the single-differential radiation cross section,

$$
\begin{aligned}
\frac{d \chi}{d \hbar \omega}= & 8 \alpha Z_{t}^{2} Z^{2} r_{p}^{2} \\
& \times\left(\left(Z M_{p} / M\right)^{2}\left[G\left(2 \gamma \omega_{1} / \omega\right)-G(1)\right] \Theta\left(2 \gamma \omega_{1}-\omega\right)\right. \\
& +\left[G\left(2 \gamma \omega_{2} / \omega\right)-G\left(\max \left\{1,2 \gamma \omega_{1} / \omega\right\}\right)\right] \Theta\left(2 \gamma \omega_{2}-\omega\right) \\
& \left.+\left[G\left(\left(\gamma \omega_{2} / \omega\right)^{2}\right)-G\left(2 \gamma \omega_{2} / \omega\right)\right] \Theta\left(\gamma \omega_{2} / 2-\omega\right)\right),
\end{aligned}
$$

where the function $G$ is defined as

$$
G=\int d t \frac{t^{2}-2 t+2}{t^{4}} \ln \left(\frac{C}{\xi^{\prime}}\right), \quad t=1+\gamma^{2} \theta^{2},
$$

with $C / \xi^{\prime}$ given by Eq. (20). For a neutral target atom (screened exponentially at the Thomas-Fermi distance), $G$ assumes the

$$
\sin ^{2} \frac{\psi^{\prime}}{2} \simeq \frac{1}{1+\gamma^{2} \theta^{2}}
$$

$\left(\theta^{\prime}=\pi-\psi^{\prime}\right)$.

\section{Radiation cross section}

Combining the results (6), (7), (10)-(12), and (17), we get

$$
\frac{d^{2} \chi}{d \hbar \omega d \Omega}=\frac{16}{3} \alpha Z_{t}^{2} Z^{2} r_{p}^{2} \frac{3 \gamma^{2}\left(1+\gamma^{4} \theta^{4}\right)}{2 \pi\left(1+\gamma^{2} \theta^{2}\right)^{4}} \ln \left(\frac{C}{\xi^{\prime}}\right) \Xi,
$$

where, according to (9) and (16),

$$
\begin{aligned}
& \frac{C}{\xi^{\prime}}=\frac{2 \gamma^{2} c C}{\omega R_{\Sigma}\left(1+\gamma^{2} \theta^{2}\right)}
\end{aligned}
$$

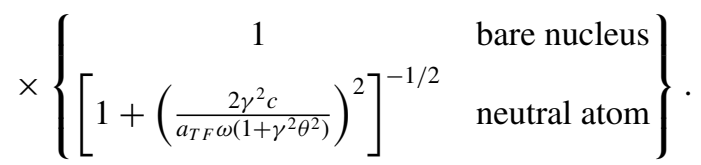

The quantity $\Xi$ is the factor in curly brackets in the scattering cross section [Eqs. (6) and (7)] with frequency and emission angle expressed by their laboratory values according to Eqs. (16) and (18).

For the hypothetical scattering cross section (6), we have

$$
\left.\begin{array}{l}
\left(1+\gamma^{2} \theta^{2}\right) \omega<2 \gamma \omega_{1} \\
2 \gamma \omega_{1}<\left(1+\gamma^{2} \theta^{2}\right) \omega<2 \gamma \omega_{2} \\
2 \gamma \omega_{2}<\left(1+\gamma^{2} \theta^{2}\right) \omega
\end{array}\right\}
$$

form

$$
\begin{aligned}
G(t)= & \frac{2 / 3-b^{2}}{b^{3}}\left[\tan ^{-1}\left(\frac{t}{b}\right)-\frac{\pi}{2}\right]+\frac{1}{2 b^{2}} \ln \left(\frac{t^{2}}{t^{2}+b^{2}}\right) \\
& +\frac{2}{3 b^{2} t}-\frac{t^{2}-t+2 / 3}{2 t^{3}} \ln \left(\frac{a^{2}}{t^{2}+b^{2}}\right)
\end{aligned}
$$

with parameters defined as

$$
a=\frac{2 \gamma^{2} c C}{\omega R_{\Sigma}}, \quad b=\frac{2 \gamma^{2} c}{a_{T F} \omega} .
$$

For a bare target nucleus, $G$ reduces to

$$
G_{0}(t)=\frac{1}{t}-\frac{1}{2 t^{2}}+\frac{2}{9 t^{3}}-\frac{t^{2}-t+2 / 3}{t^{3}} \ln \left(\frac{a}{t}\right) .
$$

The calculation of the radiation cross section for the realistic scattering cross section (7) proceeds in analogy to the calculation for the hypothetical case. Introducing the notation

$$
\begin{aligned}
& H\left(t_{\uparrow} ; t_{\downarrow}\right) \\
& =0.793 \int_{t_{\downarrow}}^{t_{\uparrow}} d t \frac{t^{2}-2 t+2}{\left(t^{2}-\left(2 \gamma E_{m} / \hbar \omega\right)^{2}\right)^{2}+t^{2}(2 \gamma \Gamma / \hbar \omega)^{2}} \ln \left(\frac{C}{\xi^{\prime}}\right)
\end{aligned}
$$


and

$$
\begin{aligned}
I\left(t_{\downarrow}\right)= & 1.93 \int_{t_{\downarrow}}^{“ \infty^{\prime \prime}} d t \frac{t^{2}-2 t+2}{t^{4}} \ln \left(\frac{C}{\xi^{\prime}}\right) \\
& \times \exp \left[-\epsilon \hbar \tilde{\omega}_{2}\left(\frac{\omega}{2 \gamma \tilde{\omega}_{2}}-\frac{1}{t}\right)\right],
\end{aligned}
$$

where $t \equiv 1+\gamma^{2} \theta^{2}$ as in Eq. (23) and $C / \xi^{\prime}$ is given by Eq. (20), we get

$$
\begin{aligned}
\frac{d \chi}{d \hbar \omega}= & 8 \alpha Z_{t}^{2} Z^{2} r_{p}^{2} \\
& \times\left(\left(Z M_{p} / M\right)^{2}\left[G\left(2 \gamma \omega_{1} / \omega\right)-G(1)\right] \Theta\left(2 \gamma \omega_{1}-\omega\right)\right. \\
& +H\left(2 \gamma \tilde{\omega}_{2} / \omega ; \max \left\{1,2 \gamma \omega_{1} / \omega\right\}\right) \Theta\left(2 \gamma \tilde{\omega}_{2}-\omega\right) \\
& \left.+I\left(\max \left\{1,2 \gamma \tilde{\omega}_{2} / \omega\right\}\right)\right) .
\end{aligned}
$$

Actual values of $H$ and $I$ are obtained by numerical integration. For the case of bare target nuclei, it is possible to write up a closed expression for the latter (containing the exponential integral Ei). The somewhat unconventional notation for the upper limit of integration in Eq. (28) signifies that this limit, although very large, is not formally infinite since the angle is limited to $\pi$. Furthermore, since the asymptotic form of the virtual-photon spectrum has been applied, integration should only extend up to the zero point of the logarithmic factor in the integrand.

\section{E. Bremsstrahlung spectra}

Figure 1 shows the hypothetical bremsstrahlung spectrum obtained from Eqs. (22), (24), and (25) for a bare lead nucleus impinging on a lead target at a $\gamma$ value of 170 . The energies characterizing the photon scattering in $\mathcal{R}$ are

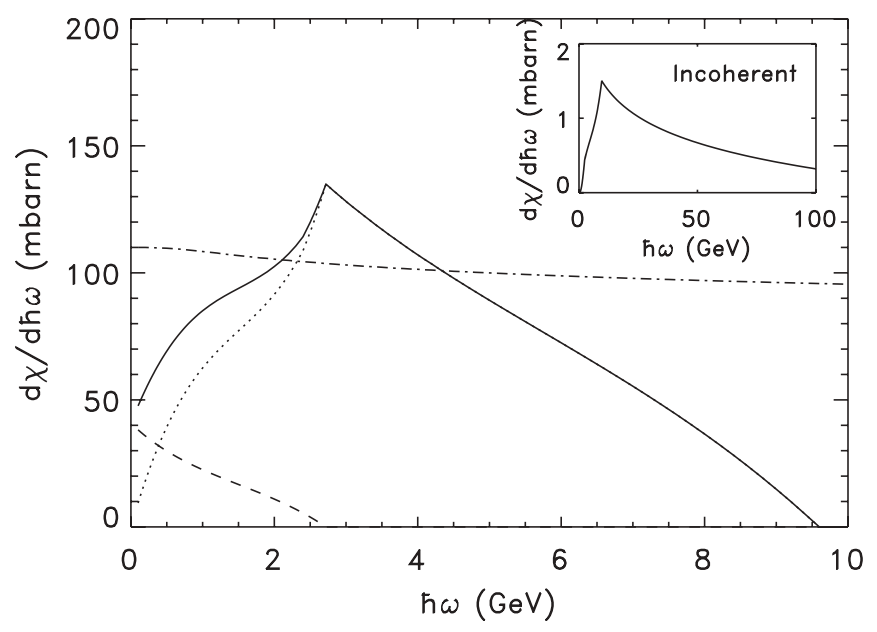

FIG. 1. Radiation cross section for noncontact encounters of bare lead ions ( $\gamma=170)$ with neutral lead atoms (at rest) obtained with the hypothetical scattering cross section (6). The dashed curve shows the part of the spectrum where the projectile acts as a rigid structureless charged particle [upper line in Eq. (6)], the dotted curve corresponds to coherent action of $Z$ quasifree protons [two lower lines in Eq. (6)], and the solid curve is the sum of these two contributions. The chained curve displays the result (1) with the logarithmic factor given by (3). The insert shows the radiation cross section corresponding to incoherent action of $Z$ quasifree protons in noncontact collisions, Subsec. III G.

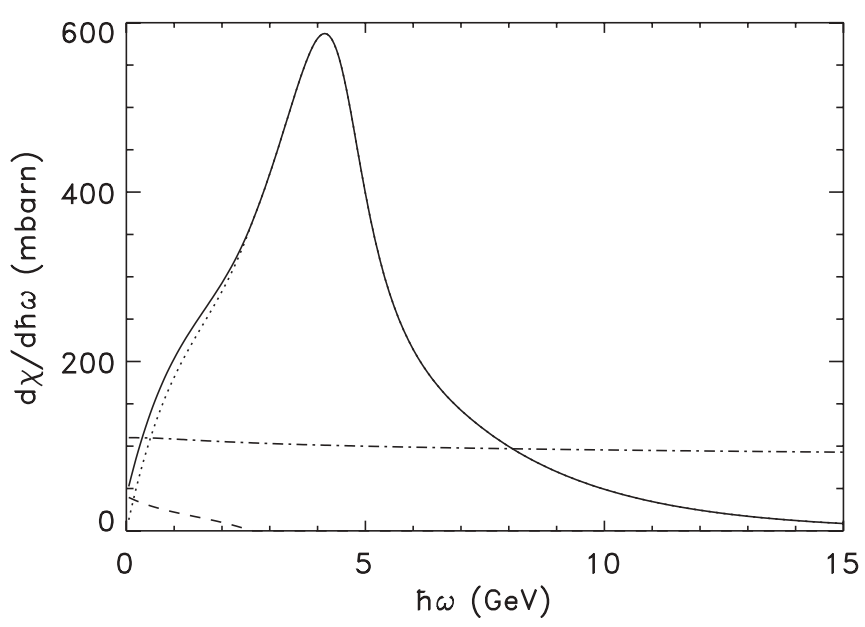

FIG. 2. Radiation cross section for noncontact encounters of bare lead ions $(\gamma=170)$ with neutral lead atoms (at rest) obtained with the representation (7) of the cross section for elastic photon scattering on ${ }^{208} \mathrm{~Pb}$ nuclei. The solid curve shows the full bremsstrahlung spectrum, the dashed curve displays the contribution from scattering events where the projectile acts as a rigid structureless object $\left(\omega^{\prime}<\omega_{1}\right)$, and the dotted curve shows the contribution originating from elastic scattering at frequencies beyond $\omega_{1}$ in the projectile rest frame. The dash-dotted curve is identical to the chained curve in Fig. 1.

chosen as $\hbar \omega_{1}=8 \mathrm{MeV}$ and $\hbar \omega_{2}=\hbar c / R=28.2 \mathrm{MeV}$. The spectrum peaks at $2 \gamma \hbar \omega_{1}=2.72 \mathrm{GeV}$ and terminates at $2 \gamma \hbar \omega_{2}=9.60 \mathrm{GeV}$. The somewhat unusual shape of the peak is due to the discontinuity of the scattering cross section (6) at $\omega_{1}$ (a jump by a factor of 6 occurs here for lead). The figure further shows that processes where the projectile acts as a structureless rigid object [first component of Eq. (22)] contribute only modestly to the radiation cross section except for energies tending to zero where it gives the sole contribution. By far, the major part of the radiation cross section derives from coherent scattering of virtual photons on $Z$ quasifree protons [last two components of Eq. (22)] at essentially all finite photon energies. The computed bremsstrahlung spectrum is less, for most energies much less, than the result (1) pertaining to a pointlike and structureless particle of the same charge and mass as the projectile nucleus except in a very narrow region near the peak where the computed spectrum slightly overshoots (1).

Figure 2 shows the realistic bremsstrahlung spectrum obtained from Eq. (29) with $G, H$, and $I$ given by Eqs. (24), (25), (27), and (28) for a bare lead nucleus impinging on a lead target at a $\gamma$ value of 170 . The spectrum shows features similar to the spectrum based on the hypothetical scattering cross section (Fig. 1). But due to the giant dipole resonance in the scattering cross section, the peak value is now more than four times higher than the maximum in Fig. 1, the peak position is shifted upward in energy by roughly $50 \%$, and the peak shows a smoother behavior. Due to the strong GDR, the contribution from events where the projectile acts as a rigid and structureless scatterer is of even less importance relatively than in the hypothetical case. Also due to the GDR, the bremsstrahlung spectrum is stronger than that for a hypothetical point nucleus (of the same charge and mass as the actual projectile) up to, about, $8 \mathrm{GeV}$. Due to the exponential depletion of the scattering 


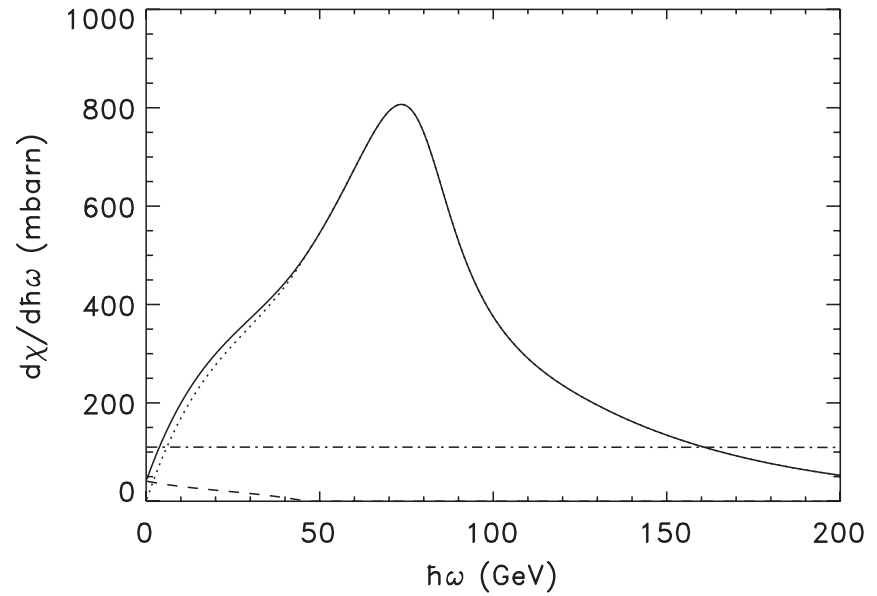

FIG. 3. As Fig. 2 but with $\gamma=3000$.

cross section (7) at high photon frequencies, the spectrum falls gradually to zero rather than abruptly as the spectrum of Fig. 1.

Figure 3 shows the bremsstrahlung spectrum, again obtained from Eq. (29), for a bare lead nucleus impinging on a lead target at an energy as that foreseen for CERN's Large Hadron Collider, $\gamma=3000$. The spectrum shows the same features as the spectrum for $\gamma=170$ (Fig. 2); only the energies are shifted upward in proportion to $\gamma$ and the peak value is $37 \%$ higher.

Figure 4 demonstrates the variation with primary energy quite clearly: By plotting as a function of photon energy divided by the $\gamma$ value of the projectile, spectra for different projectile energies fall on top of each other. The peak value increases with $\gamma$ but saturates due to screening. The peak position is slightly below twice the peak position of the giant dipole resonance, $2 E_{m}=0.0274 \mathrm{GeV}$. The two other characteristic energies, $2 \hbar \omega_{1}=0.0154 \mathrm{GeV}$ and $2 \hbar \tilde{\omega}_{2}=0.044 \mathrm{GeV}$, are positioned on opposite sides of the peak.

Atomic screening becomes increasingly important as the projectile energy is raised. For bare lead ions at $\gamma=170$, the

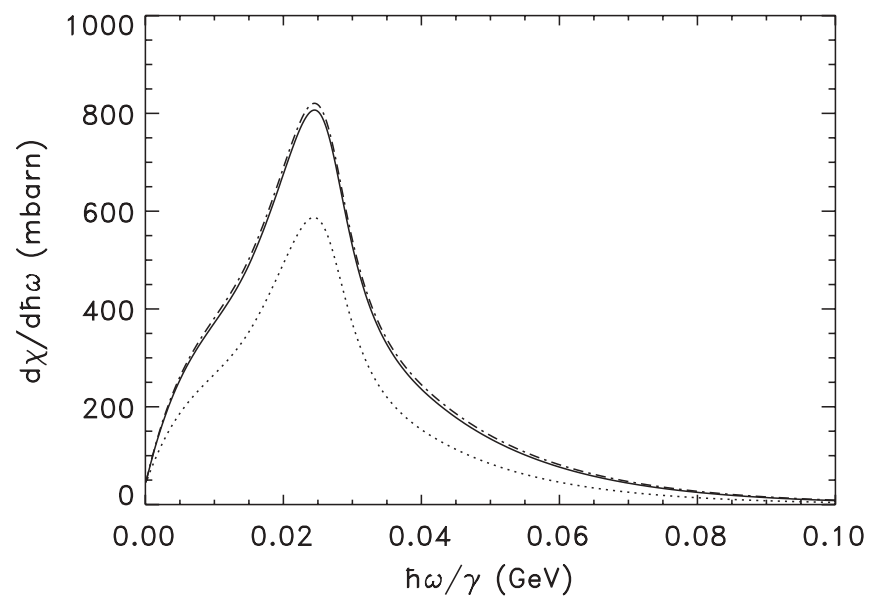

FIG. 4. Radiation cross section for noncontact encounters of bare lead ions with neutral lead atoms at rest as a function of photon energy divided by $\gamma$. Spectra for three different values of $\gamma$ are displayed: $\gamma=3000$ full-drawn curve, $\gamma=170$ dotted, and $\gamma=$ 100000 chained.

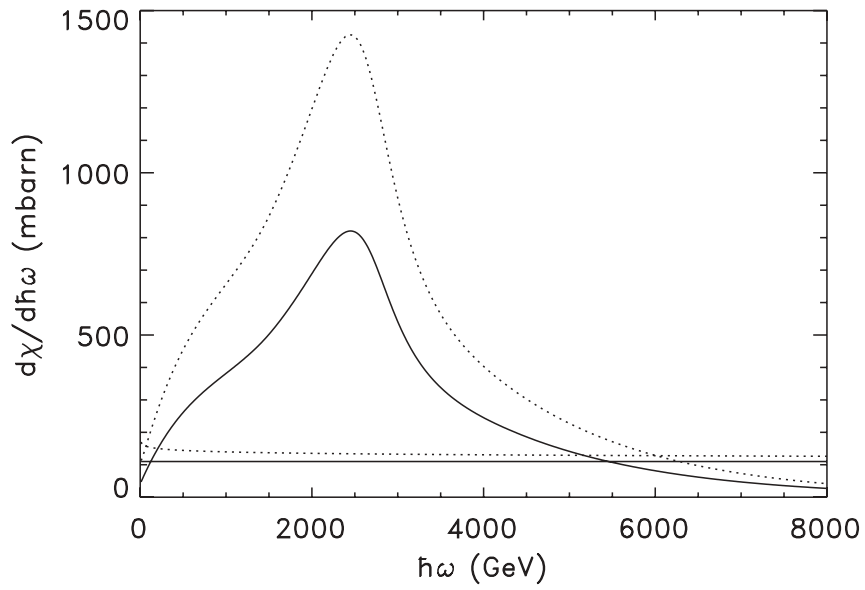

FIG. 5. Effect of screening on bremsstrahlung at high projectile energy. A bare lead ion is incident on a lead target at $\gamma=100000$. The full-drawn peaked spectrum is obtained by the same procedure as the full-drawn curve in Fig. 2 ("screening included"). The dotted peaked spectrum shows the result of the same calculation if the neutral target atom is replaced by a bare ion ("no screening"). The two flat curves display the point-nucleus result (1) with (full-drawn) and without (dotted) target screening.

difference in the bremsstrahlung intensity for an atomic lead target and a target consisting of bare lead ions is less than $1 \%$ for photon energies above $1 \mathrm{GeV}$. The difference increases with decreasing photon energy, but at the lower crossing point with the result (1) pertaining to a pointlike projectile $(0.3 \mathrm{GeV})$, the difference still only amounts to $5 \%$. Figure 5 shows the results for the same collision systems when the $\gamma$ value of the projectile is raised to a very high value, $\gamma=100000$. Obviously screening has a major influence on bremsstrahlung throughout the spectrum at this energy.

\section{F. Energy loss}

The stopping power due to bremsstrahlung emission, that is, the radiative energy loss per unit penetrated depth, is obtained as the atomic density $N$ of the target times the integral of the radiation cross section [Eq. (29) with the definitions (24), (25), (27), and (28)]

$$
-\frac{d E}{d x}=N \int d \hbar \omega \frac{d \chi}{d \hbar \omega} .
$$

Figure 6 shows a plot of this stopping power divided by the total projectile energy $E=\gamma M c^{2}$ as a function of $\gamma$ for lead ions penetrating a lead target. Due to screening, the relative energy loss saturates at high projectile energies at a value of $N \times 0.119$ mbarn $\left(3.91 \times 10^{-6} \mathrm{~cm}^{-1}\right)$. In Ref. [4], we gave a rough estimate of the radiative energy loss. For the considered collision system, this estimate, Eq. (7) in Ref. [4], comes out at around $2 / 3$ of the result shown in Fig. 6 for bare target nuclei. (In view of the roughness of the estimate, it should be considered accidental that it hits this close to the actual value of the energy loss.)

The cross section (1) pertaining to a pointlike and structureless lead nucleus is around 0.1 barn for photon energies all way up to the primary (kinetic) energy. Hence the actual radiative stopping power (Fig. 6) is down by three orders of 


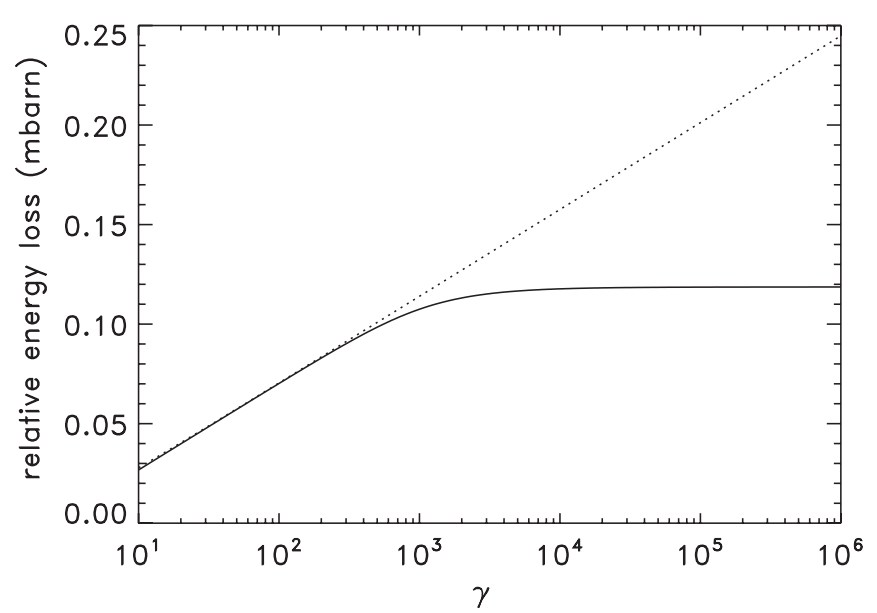

FIG. 6. Energy loss through bremsstrahlung emission for a lead ion penetrating a lead target. The figure shows $-N^{-1} E^{-1} d E / d x$ as a function of the $\gamma$ value of the projectile. The full-drawn curve displays the result for an atomic target. The dotted curve pertains to the hypothetical case of a target consisting of bare lead ions (no screening). To get the fractional energy loss $-E^{-1} d E / d x$ per $\mathrm{cm}$, the ordinate in the figure should be multiplied by $3.30 \times 10^{-5}$.

magnitude compared with what one would get by assuming the projectile to radiate as a pointlike and structureless object.

In Ref. [4], we produced a rough estimate showing that bremsstrahlung emission is of considerably less importance for the slowing down of relativistic bare heavy ions in matter than electron-positron pair creation. We may now quantify that for our standard collision system. Neglecting the small contribution from pair creation on atomic electrons, the stopping power due to pair production is

$$
\left(-\frac{d E}{d x}\right)_{P P}=\pi Z^{2} Z_{t}^{2} \alpha^{2} r_{e}^{2} N \gamma m c^{2} \Lambda,
$$

where $m$ is the electron mass and $r_{e} \equiv e^{2} / m c^{2}$ the classical electron radius. The logarithmic factor $\Lambda$ is approximately given as

$$
\Lambda=\frac{19}{9} \ln \frac{183 Z_{t}^{-1 / 3}}{1+4 e^{11 / 6} 183 Z_{t}^{-1 / 3} / \gamma} ;
$$

see [4]. For bare lead ions penetrating a lead target, Eqs. (31) and (32) produce stopping powers which are higher than the radiative stopping power computed above by factors ranging from 74 to 105 for $\gamma$ values ranging from 170 to 100000 . That is, pair production overshoots bremsstrahlung in the energy-loss process for relativistic heavy ions by two orders of magnitude.

Let us close the discussion by comparing the radiative energy loss to the stopping due to atomic excitation and ionization (the "electronic energy loss") which brings the major contribution to the stopping at nonrelativistic as well as moderately relativistic energies. The electronic stopping power is given as

$$
\left(-\frac{d E}{d x}\right)_{\text {electr }}=\frac{4 \pi Z^{2} e^{4}}{m v^{2}} N Z_{t} L,
$$

where $N Z_{t}=n$ is the average density of target electrons. For energies sufficiently high that the density effect is in full

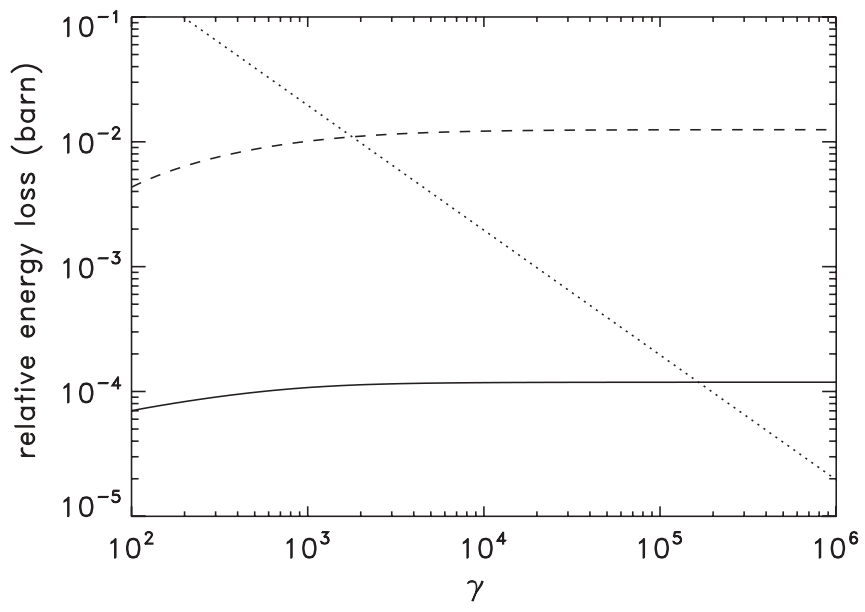

FIG. 7. Energy loss of bare lead ions in a lead target. The fulldrawn curve is the same as in Fig. 6, the dashed curve shows the energy loss through electron-positron pair creation [Eqs. (31) and (32)], and the dotted curve shows the ionization energy loss [Eq. (33) with the high-energy approximation Eq. (34)].

action and close collisions are limited by the finite size of the projectile nucleus, the logarithmic factor $L$ takes the simple form [11]

$$
L \rightarrow \ln \left(1.62 c / R \omega_{\mathrm{pl}}\right),
$$

where $\omega_{\mathrm{pl}}=\sqrt{4 \pi n e^{2} / m}$ is the plasma frequency of the target. For heavy projectiles in condensed matter, Eq. (34) applies quite accurately for $\gamma$ values beyond 100 . For bare ${ }^{208} \mathrm{~Pb}$ ions penetrating a lead target, Eqs. (33) and (34) give a relative energy loss of $N \times 19.6 / \gamma$ barn. The electronic and the radiative losses become equal at $\gamma=1.65 \times 10^{5}$ beyond which value the radiative loss is the larger of the two. The electronic and the pair-production losses become equal at $\gamma=1790$ (or, if production on atomic electrons is included, at $\gamma=1730$ [3]) beyond which value pair-production losses dominate. Figure 7 gives an overview of the magnitude of the energy-loss mechanisms.

\section{G. Incoherent scattering}

Bremsstrahlung is emitted as a result of coherent scattering of virtual photons of the target on the projectile in noncontact collision. Incoherent scattering on single protons in the projectile nucleus generally results in breakup. Let us terminate the study of noncontact processes by an estimate of radiation emitted as a result of incoherent photon scattering.

Roughly, incoherent scattering requires $\omega^{\prime}>\omega_{2}$, and the cross section is given approximately by an expression similar to the last line in Eq. (6) except that $Z^{2}$ has to be replaced by $Z$ and the angular restriction (the argument of the Heaviside function) is the opposite. Going through the same steps as in Sec. IIID, we end up with the following estimate for the incoherent radiation cross section:

$$
\frac{d \chi}{d \hbar \omega}=8 \alpha Z_{t}^{2} Z r_{p}^{2}\left[-G\left(\max \left\{1,2 \gamma \omega_{2} / \omega,\left(\gamma \omega_{2} / \omega\right)^{2}\right\}\right)\right],
$$

where $G$ is given by Eq. (24) or, for a bare target nucleus, by Eq. (26). In the derivation of Eq. (35), we have neglected the value of $G$ at the upper limit of integration compared to that 
at the lower limit. This requires $\omega \gg \omega_{2}$, which is of course always fulfilled for $\mathrm{GeV}$ photon energies. For bare nuclei and $\omega>2 \gamma \omega_{2}$, the incoherent radiation cross section reduces to

$$
\frac{d \chi}{d \hbar \omega}=\frac{16}{3} \alpha Z_{t}^{2} Z r_{p}^{2}\left(\ln \frac{2 \gamma^{2} c C}{\omega R_{\Sigma}}-\frac{13}{12}\right) .
$$

As opposed to the coherent case incoherent scattering is not limited to rest-frame energies of a few times $\hbar \omega_{2}$, and hence, the incoherent radiation is not limited to a few times $2 \gamma \hbar \omega_{2}$ but extends to much higher energies. Despite this, we have used the asymptotic expression (10) for the virtual photon spectrum to obtain the expressions (35) and (36). In the main, this gives a quite reasonable estimate, but the expressions should of course not be used where they produce negative values and the depletion with increasing energy near their zero point is obviously less abrupt than Eqs. (35) and (36) predict. The insert in Fig. 1 shows the estimate (35) of the incoherent radiation cross section. It is much lower than the bremsstrahlung in the region where the latter has its support but extends to much higher energies.

\section{CONTACT COLLISIONS}

In Secs. III A-IIIF, we considered the bremsstrahlung emitted in collisions between a relativistic bare heavy ion and a stationary target atom at impact parameters larger than the sum of the nuclear radii. This is our main focus. For comparison, let us now turn to the electromagnetic radiation emitted in collisions with nuclear contact where the collision partners break up. We aim for the characteristics and level of the radiation, particularly in the region where bremsstrahlung is appreciable, and consider a rough estimate sufficient.

Initially we proceed along the lines laid out by Jeon et al. [12]. Although they present a relatively simple calculation, we note that a later study [13] based on a microscopic transport model (the so-called ultrarelativistic quantum molecular dynamics model) produces results which agree with the outcome of Eq. (11) in Ref. [12] within 20-30\% for those angles which contribute the major part of the total cross section. The calculation is based on classical electrodynamics according to which the radiated intensity per frequency $\omega$ and per solid angle $\Omega$ may be expressed in terms of the electromagnetic current density $\mathbf{J}$ of the source as

$$
\begin{gathered}
\frac{d^{2} I}{d \omega d \Omega}=\frac{\omega^{2}}{4 \pi^{2} c^{3}}|\mathbf{A}(\mathbf{n}, \omega)|^{2}, \\
\mathbf{A}(\mathbf{n}, \omega) \equiv \int d t \int d^{3} r \mathbf{n} \times[\mathbf{n} \times \mathbf{J}] e^{i \omega(t-\mathbf{n} \cdot \mathbf{r} / c)},
\end{gathered}
$$

where $\mathbf{n}$ is the direction of emission; see [6].

\section{A. Instantaneous interaction}

We consider a central collision between two identical nuclei of spherical shape, but as opposed to the situation described in Ref. [12], one is originally at rest in the laboratory. The incoming projectile is moving along the $z$ axis in the positive direction at speed $v_{0}$ aiming at the target at rest at the origin. (In this section, subscript 0 is included on the initial projectile velocity to distinguish it from the velocities of the reaction products.) The collision happens instantaneously at time zero, $t=0$, at least in our first approach. Before the collision, the electromagnetic current density hence assumes the form

$$
\mathbf{J}=\sigma_{P}\left(r_{\perp}\right) v_{0} \delta\left(z-v_{0} t\right) \hat{\mathbf{z}}, \quad t<0,
$$

where $\hat{\mathbf{z}}$ is a unit vector along the $z$ axis in the positive direction and $\sigma_{P}\left(r_{\perp}\right)$ is the charge density of the projectile integrated along $z$ at fixed transverse position $r_{\perp}=\sqrt{x^{2}+y^{2}}$ (Cartesian coordinates). After the collision, all fragments are assumed to move parallel to the $z$ axis with a distribution in the longitudinal velocity $v$. Following Jeon et al. [12], we introduce the rapidity $\Upsilon$ defined by the relation

$$
v / c=\tanh \Upsilon
$$

where $c$ is the speed of light. The distribution in velocity translates into a distribution $\rho$ in rapidity,

$$
\int_{-\infty}^{\infty} d \Upsilon \rho\left(r_{\perp}, \Upsilon\right)=1,
$$

and the current density after the collision may hence be written as

$$
\mathbf{J}=\sigma_{F}\left(r_{\perp}\right) \int_{-\infty}^{\infty} d \Upsilon \rho\left(r_{\perp}, \Upsilon\right) v(\Upsilon) \delta(z-v(\Upsilon) t) \hat{\mathbf{z}}, \quad t>0
$$

No redistribution of nuclear matter is assumed to take place in transverse space which implies that $\sigma_{F}\left(r_{\perp}\right)=2 \sigma_{P}\left(r_{\perp}\right)$.

Since the current density in our model always is directed along the $z$ axis, the vector $\mathbf{n} \times[\mathbf{n} \times \mathbf{J}]$ has a fixed direction to which we assign the unit vector $\mathbf{e}_{A}$. Introducing the emission angle $\theta$ and the direction of the $x$ axis through $\mathbf{n} \equiv$ $(\sin \theta, 0, \cos \theta)$, we have $\mathbf{n} \times[\mathbf{n} \times \mathbf{J}]=\sin \theta J \mathbf{e}_{A}$. Expression (38) for $\mathbf{A}$ then reduces to

$\mathbf{A}(\mathbf{n}, \omega)=\mathbf{e}_{A} \sin \theta \int d t \int d^{3} r J(\mathbf{r}, t) e^{i \omega[t-(x \sin \theta+z \cos \theta) / c]}$.

The delta function appearing in the expressions (39) and (42) for $J$ takes care of the integral over $z$. In turn, the integration over time may be performed. Since the acceleration vanishes outside a time window of (any) finite length, the evaluation requires use of a standard trick (see, e.g., Jackson [6]): The integrand is multiplied by a convergence factor $e^{-\epsilon|t|}$, the integral is subsequently evaluated, and the answer is finally taken in the limit $\epsilon \rightarrow 0$. The result is

$$
\begin{aligned}
\mathbf{A}(\mathbf{n}, \omega)= & \mathbf{e}_{A} \frac{\sin \theta}{i \omega} \int d^{2} r_{\perp} e^{-i \omega x \sin \theta / c} \sigma_{P}\left(r_{\perp}\right) \\
& \times\left[\frac{v_{0}}{1-\beta \cos \theta}-2 \int_{-\infty}^{\infty} d \Upsilon \rho\left(r_{\perp}, \Upsilon\right) \frac{v(\Upsilon)}{1-\beta_{\Upsilon} \cos \theta}\right],
\end{aligned}
$$

where $\beta \equiv v_{0} / c$ and $\beta_{\Upsilon} \equiv v(\Upsilon) / c$. The distribution in rapidity was computed in Ref. [12] in a microscopic model (different from that used later in Ref. [13]) for two identical gold nuclei colliding head on at an energy of $100 \mathrm{GeV} / \mathrm{n}$ (equal but opposite velocities). The distribution was found to be roughly flat overall. Furthermore, the radiation corresponding to a flat distribution was found to be quite close to that corresponding to the computed distribution. For our purpose of getting a relatively rough idea of the radiation emitted in contact 
collisions, we shall hence assume a similar flat distribution in $\Upsilon$ and $r_{\perp}$ for the fixed-target case; that is,

$$
\rho=1 / \Upsilon_{0} \quad \text { for } \quad 0 \leqslant \Upsilon \leqslant \Upsilon_{0} \quad \text { and } \quad r_{\perp}<R,
$$

where $R$ is the nuclear radius, and $\rho=0$ otherwise. The maximum rapidity $\Upsilon_{0}$ corresponds to the velocity $v_{0}$ of the incoming ion,

$$
\Upsilon_{0}=\tanh ^{-1} \beta=\ln [(1+\beta) \gamma] \simeq \ln (2 \gamma),
$$

where $\gamma \equiv 1 / \sqrt{1-\beta^{2}}$ is the Lorentz factor of the projectile. With $\rho$ independent of $r_{\perp}$ (inside $R$ ), the square-bracket factor in Eq. (44) may be taken outside the integral and the remaining integral over transverse coordinates reduces to $Z e F(\omega \sin \theta / c)$, where $Z e$ is the nuclear charge and $F$ is the form factor defined as

$$
F(q)=(Z e)^{-1} \int d^{3} r \rho_{N}(\mathbf{r}) e^{i \mathbf{q} \cdot \mathbf{r}},
$$

where $\rho_{N}$ is the nuclear charge density. Finally, performing the integration over rapidity and inserting the resulting expression for $\mathbf{A}$ into Eq. (37) leads to the differential intensity

$$
\begin{aligned}
& \frac{d^{2} I}{d \hbar \omega d \Omega}=\frac{\alpha}{4 \pi^{2}} \sin ^{2} \theta Z^{2}|F(\omega \sin \theta / c)|^{2} S^{2} \\
S \equiv & 2 \frac{\cos \theta}{\sin ^{2} \theta}+\frac{2}{\Upsilon_{0} \sin ^{2} \theta} \ln (\gamma(1-\beta \cos \theta))-\frac{\beta}{1-\beta \cos \theta} .
\end{aligned}
$$

The introduction of $\hbar$ has produced a dimensionless result. Equations (48) and (49) represent a transcription to the fixedtarget case of the final Eq. (11) in Ref. [12]; the modification appears entirely in the factor $S^{2}$.

\section{B. Characteristic angles and radiation cross section}

The differential intensity [Eqs. (48) and (49)] vanishes at $\theta=0$ and peaks at a small angle for high values of $\gamma$. For the moment, let us neglect the form factor (that is, we put $F=1$, which in any case is true by definition in the limit $\theta=0$ ). A small-angle expansion gives

$$
S \simeq \frac{2 \Upsilon_{0}^{-1} \ln \left(1+(\gamma \theta)^{2}\right)-\theta^{2}}{\theta^{2}}-\frac{2 \gamma^{2}}{1+(\gamma \theta)^{2}}, \quad \theta \ll 1 .
$$

This shows that the characteristic angle of emission is $1 / \gamma$,

$$
\theta \sim 1 / \gamma
$$

It may further be noted that $S$ changes sign at an angle $\theta_{0}$ way beyond $1 / \gamma$. From the expansion (50) and the result (46), it is seen that

$$
\theta_{0}^{2} \simeq 2 / \gamma
$$

in the limit of high $\gamma$. With $F=1$, there is exact equipartition of the intensity at $\theta_{0}$; that is, integration over emission angles smaller and larger than $\theta_{0}$ gives identical results,

$$
\left.\frac{d I}{d \hbar \omega}\right|_{\theta<\theta_{0}}=\left.\frac{d I}{d \hbar \omega}\right|_{\theta>\theta_{0}}, \quad F=1
$$

With $F=1$, there is no dependence on emission frequency and $d I / d \hbar \omega$ shows roughly a dependence on primary energy of the form $a \ln \gamma+b$ ( $a$ and $b$ constants).

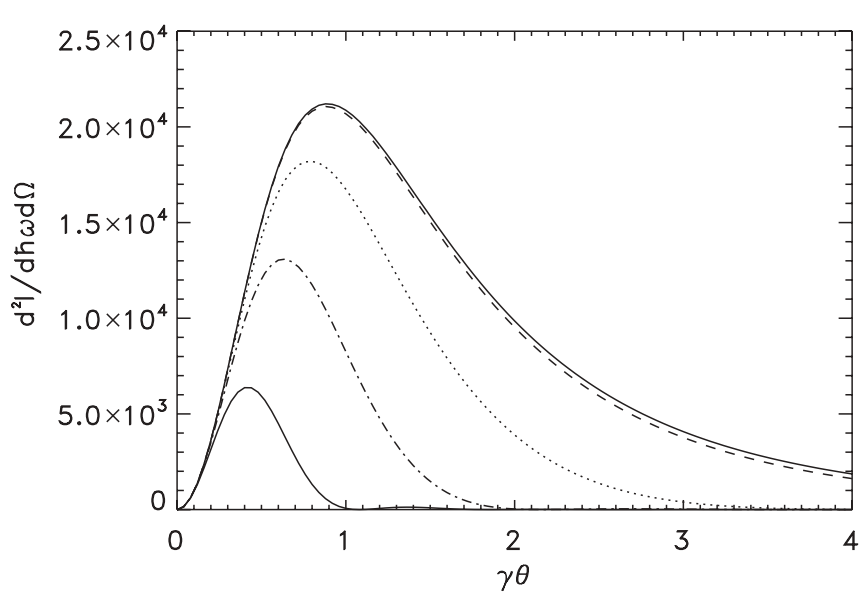

FIG. 8. Bremsstrahlung intensity in central collision between two bare lead ions as a function of emission angle. The curves correspond to photon energies of $0 \mathrm{GeV}$ (upper full drawn), $1 \mathrm{GeV}$ (dashed), $5 \mathrm{GeV}$ (dotted), $10 \mathrm{GeV}$ (chained), and $20 \mathrm{GeV}$ (lower full drawn). One ion is originally at rest, and the other is impinging at $\gamma=170$.

Inclusion of the form factor introduces a variation of the radiation intensity with frequency. Generally, $F$ causes a damping for arguments larger than, approximately, $1 / R$. Near the peak in the differential spectrum, where $\theta \sim 1 / \gamma$, this implies damping for

$$
\hbar \omega \gtrsim 0.2 \mathrm{GeV} \mathrm{fm} \times \gamma / R
$$

For lead ions at $\gamma=170$, this estimate reads as $\hbar \omega \gtrsim 5 \mathrm{GeV}$. As to the specific expression for $F$, we shall choose that pertaining to a homogeneously charged sphere of radius $R$,

$$
F(q)=\frac{3}{(q R)^{2}}\left[\frac{\sin (q R)}{q R}-\cos (q R)\right] .
$$

Figure 8 displays the double-differential spectrum [Eqs. (48) and (49)] with the form factor given by Eq. (55) as a function of emission angle for different photon energies for our standard case. The curve for vanishing photon energy corresponds to $F=1$. The remaining curves show how damping increases with $\hbar \omega$ and confirm the estimate (54). It should be noted that while the curve for $\hbar \omega=1 \mathrm{GeV}$ shows rather limited damping in the region around the characteristic emission angle displayed in Fig. 8, the integrated intensity $d I / d \hbar \omega$ is actually reduced to $45 \%$ of its value at $\hbar \omega=0 \mathrm{GeV}$.

Integration of the expression (48) over angles gives the intensity $d I / d \hbar \omega$ for a collision at impact parameter $b=0$. We define the radiation cross section as

$$
\frac{d \chi}{d \hbar \omega}=\int d^{2} b \frac{d I}{d \hbar \omega}(b) .
$$

As a rough model for the radiation cross section pertaining to collisions with nuclear contact, we shall assume that the variation of intensity with impact parameter is given by the variation in overlap area between two coplanar circular disks of radius $R$ with interspacing between centers. Equation (56) 


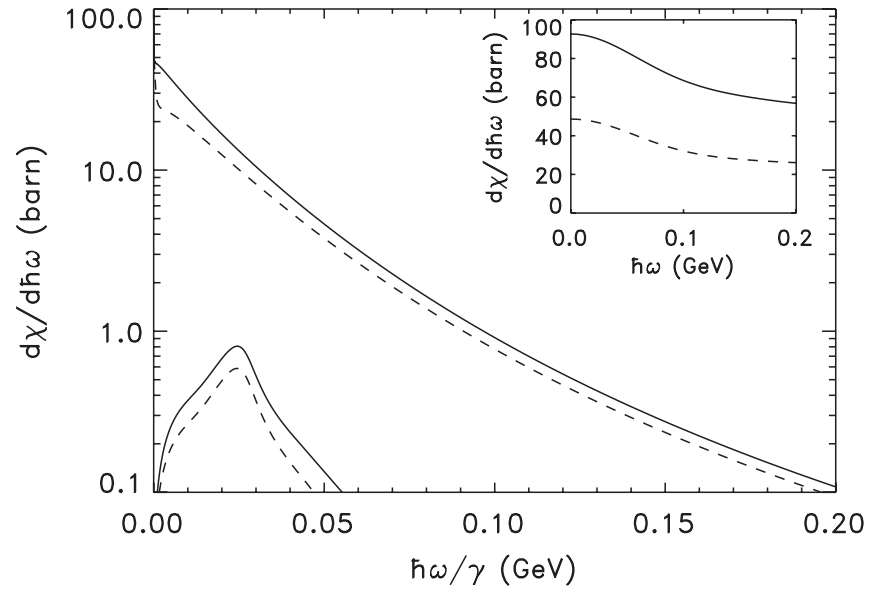

FIG. 9. Radiation cross section for collisions with nuclear contact as a function of emission energy. The collision partners are lead nuclei, one is originally at rest, and the other is impinging at $\gamma=3000$ (solid line) or $\gamma=170$ (dashed line). The abscissa in the main plot is photon energy divided by $\gamma$. The insert shows the variation at very small emission energies. Curves in the lower left of the figure show the bremsstrahlung spectra for the same two values of $\gamma$ (repeated from Fig. 4).

then reduces simply to

$$
\begin{aligned}
\frac{d \chi}{d \hbar \omega} & =\pi R^{2} \frac{d I}{d \hbar \omega}(0) \\
& =\frac{\alpha}{4 \pi} R^{2} Z^{2} \int_{4 \pi} d \Omega \sin ^{2} \theta|F(\omega \sin \theta / c)|^{2} S^{2}
\end{aligned}
$$

where $R$ is defined in Eq. (4) and $S$ is given in Eq. (49). For lead ions in a lead target, the factor in front of the integral in Eq. (57) is 1.9 barn. Figure 9 shows the variation of the radiation cross section (57) with photon energy for two largely different values of the Lorentz factor. The cross section is well above the reference electromagnetic radiation cross section (1) [0.110 barn for complete screening, see discussion after Eq. (3)] up to, roughly, $0.2 \gamma \mathrm{GeV}$. Generally, we observe a near-exponential decrease with increasing photon energy. By plotting the cross section as a function of the reduced variable $\hbar \omega / \gamma$ nearly identical results, up to a $\omega$-independent factor, are obtained for the two $\gamma$ values over the major part of the photon-energy range [cf. Eq. (54)]. At very low energies, a rapid variation is superimposed. For both $\gamma$ values, the radiation cross section is reduced to two thirds of its maximum value (obtained at $\hbar \omega=0$ ) over the first $100 \mathrm{MeV}$ or so (see insert in Fig. 9). This rapid reduction reflects the equipartition (53) for $F=1$ which, in view of (52), implies that half of the intensity is emitted at angles much larger than $1 / \gamma$ for $F=1$. The large-angle contribution is damped at much smaller photon energies than the small-angle contribution when the form factor is introduced. Figure 9 also includes the bremsstrahlung spectra from Fig. 4. Obviously, the radiation in central collisions is considerably stronger than bremsstrahlung emitted in ultraperipheral collisions, and it extends to higher photon energies.

\section{Finite collision time}

The instantaneous change of the electromagnetic current at $t=0$ assumed so far implies that the formulas quoted in the previous subsections apply to the realistic collision system only for sufficiently low frequencies. At higher frequencies, lower yields are expected [6].

As a simple model, assume that the projectile current (39) is switched off linearly with time starting at $t=-\tau$ and ending at $t=\tau$,

$$
\tau \equiv R / c,
$$

and that the current (42) due to the reaction products is switched on linearly over the same time interval. This modifies the square-bracket factor in the integrand in Eq. (44) to

$$
\begin{aligned}
{[] \rightarrow } & \frac{v_{0}}{1-\beta \cos \theta} \frac{\sin [\omega \tau(1-\beta \cos \theta)]}{\omega \tau(1-\beta \cos \theta)}-2 \int_{-\infty}^{\infty} d \Upsilon \\
& \times \rho\left(r_{\perp}, \Upsilon\right) \frac{v(\Upsilon)}{1-\beta_{\Upsilon} \cos \theta} \frac{\sin \left[\omega \tau\left(1-\beta_{\Upsilon} \cos \theta\right)\right]}{\omega \tau\left(1-\beta_{\Upsilon} \cos \theta\right)} .
\end{aligned}
$$

For $\gamma \gg 1$ and $\theta \ll 1$, the argument of the temporal damping factor in the first term in (59) may be written as

$$
\omega \tau(1-\beta \cos \theta) \simeq \frac{\omega \tau}{2 \gamma^{2}}\left[1+(\gamma \theta)^{2}\right] \simeq \frac{\omega \tau}{\gamma^{2}},
$$

where the last expression applies for typical emission angles [cf. Eq. (51)]. Temporal damping sets in when the argument (60) becomes comparable to 1 , that is, for $\omega \tau \simeq \gamma^{2}$. Here, $R$ times the argument of the nuclear form factor assumes the value

$$
\omega \tau \sin \theta \simeq \omega \tau \theta \simeq \gamma^{2} / \gamma,
$$

which is obviously much larger than 1 . Hence, the damping due to the nuclear structure is already strong at emission energies where temporal damping becomes effective. The analysis for the second term in (59) is similar leading, in place of (61), to $\omega \tau \sin \theta \simeq \gamma_{\Upsilon}^{2} / \gamma$, where $\gamma_{\Upsilon}^{2} \equiv 1 /\left(1-\beta_{\Upsilon}^{2}\right)$. Except for a narrow fraction of the rapidities of the fragments damping due to nuclear structure is again more important than temporal damping.

The bottom line is that for high values of $\gamma$, only damping due to nuclear structure needs to be considered when assessing the radiation cross section. Temporal damping plays no role in practice.

\section{CONCLUDING REMARKS-OTHER SOURCES OF RADIATION}

In Sec. III, we calculated the bremsstrahlung emitted by a relativistic heavy ion as it penetrates a substance. To compare with the outcome of an experiment, other possible sources of radiation need to be identified and eliminated or accounted for. One such source is electrons ejected from target atoms, so-called delta electrons. The energy released through emission of bremsstrahlung by these secondaries is limited by the electronic energy loss [Eqs. (33) and (34)], but unless the projectile energy is very high, this does not imply a priori that the radiation by the secondaries is small compared to the 
bremsstrahlung emitted by the projectile (cf. Fig. 7). Instead we may note that the angular characteristics of the photon emission from delta electrons is quite different from that of the heavy-ion bremsstrahlung; most delta electrons are ejected at large angles, and so is their bremsstrahlung. Furthermore, being a secondary process, the radiation yield for the delta electrons scales with the square of the target thickness, whereas the intensity of the primary bremsstrahlung scales linearly with target thickness. That is, the two contributions may be separated experimentally by varying this parameter.

Bremsstrahlung may also be emitted by a target electron during the collision with the penetrating heavy ion. The energy loss due to this higher order QED process has been evaluated by Jankus [14] (see also Refs. [1] and [2]). At the lowest energies considered here, $\gamma \sim 170$, Jankus' result is an order of magnitude higher than the energy loss due to direct bremsstrahlung by the projectile for our standard collision system. But, to quote Ref. [14], the radiation should not show a marked anisotropy and the maximum photon energy should be of the order of $0.5 \mathrm{MeV}$ except for a few hard photons close to the direction of the incident heavy particle. Besides, the calculation does not take into account the density effect and the finite nuclear size. Neglect of the latter could be an important limitation since higher order QED effects primarily belong to regions with a strong field. The finite nuclear size of the projectile moderates its field in exactly this region.

As discussed in Sec. IIIF, creation of electron-positron pairs contributes more to the energy loss of a heavy ion than bremsstrahlung. The associated radiation may hence be of concern. There are two kinds of contributions exactly as for the case of ejection of target electrons. One is radiation emitted in the pair-creation process, and the other is radiation emitted after creation by the electron and positron on their way through the rest of the target. The second kind may be extracted experimentally by varying the target thickness. (And it does of course not contribute to the energy loss of the primary particle.) Furthermore, typical energies are much smaller than the characteristic energies for the heavy-ion bremsstrahlung: According to Ref. [15], the pair-production spectrum falls off beyond $\gamma m c^{2}$ roughly as the inverse cube of the total pair energy. Hence, bremsstrahlung from the pair effectively terminates at $0.5 \gamma \mathrm{MeV}$ which is a factor of 50 below the peak in the heavy-ion bremsstrahlung spectrum which appears near $25 \gamma \mathrm{MeV}$ (cf. Fig. 4).

For a rough estimate of the importance of photon emission in the electron-positron pair-creation process, we proceed as Hencken et al. [16] right before their conclusion (cross sections given in units of the square of the Compton wavelength of the electron): Up to a numerical factor, the cross section for this type of radiation is $Z^{2} Z_{t}^{2} \alpha^{5}\left(m c^{2} / \hbar \omega\right)^{2}$. The scale for the bremsstrahlung by the heavy ion is roughly set by our Eq. (1) in the region where the radiation cross section peaks (cf. Figs. 25 ; the peak typically shoots above (1) by a factor of 6-8 or so); that is, the cross section is estimated by $Z^{4} Z_{t}^{2} \alpha^{3}(m / M)^{2}$ again up to a numerical factor. The peak in the bremsstrahlung spectra appears near $25 \gamma \mathrm{MeV}$. Using this for $\hbar \omega$ in the first estimate gives a ratio of the two of $0.07(A / \gamma Z)^{2}$ which for ${ }^{208} \mathrm{~Pb}$ reduces to $0.5 / \gamma^{2}$. For the values of $\gamma$ considered here (typically at least 170), this is clearly a very small number and photon emission in the pair-creation process may be neglected.
[Note that the numerical factor in Eq. (1) is quite large, in our case, $16 L / 3 \simeq 80$. Since the actual bremsstrahlung peak furthermore overshoots (1) by a factor of 6-8, the ratios given here may actually be expected to overestimate the relative importance of photon emission in pair creation. In any case, this type of emission is only appreciable for photons with energies near or below $m c^{2}$ which in the current context is very soft photons since their energy is way below the peak in the heavy-ion bremsstrahlung spectrum.]

\section{ACKNOWLEDGMENTS}

Thanks are due to Ulrik Uggerhøj for his continuous interest in this project and for critical reading of the manuscript. This work was supported by the Danish Natural Science Research Council.

\section{APPENDIX: VIRTUAL PHOTON INTENSITY FOR YUKAWA POTENTIAL}

An observer is stationary at a point in space. A charged object is passing by with constant speed $v$ on a straight path at impact parameter $b$. Assuming $v$ to be sufficiently close to $c$ that the effects of the longitudinal field component may be neglected, the virtual photon intensity encountered by the observer is

$$
\frac{d I}{d \omega d^{2} b}=\frac{c}{2 \pi}\left|E_{\perp}(\omega)\right|^{2}
$$

(see [6]), where $E_{\perp}(\omega)$ is the Fourier transform of the component of the electric field transverse to $\mathbf{v}$ generated by the moving object at the position of the observer at time $t$,

$$
E_{\perp}(\omega)=\frac{1}{\sqrt{2 \pi}} \int_{-\infty}^{\infty} E_{\perp}(t) e^{i \omega t} d t
$$

In its own rest frame, the object generates an exponentially screened Coulomb potential. Transforming the corresponding field to the observer's frame and expressing in observer's variables (see, e.g., [6]) gives

$$
E_{\perp}(t)=\gamma\left(-\frac{\partial}{\partial b} \frac{q}{R} e^{-R / a}\right), \quad R \equiv \sqrt{b^{2}+\gamma^{2} v^{2} t^{2}},
$$

where $q$ is the central charge of the moving object, $a$ the screening length, and $\gamma$ the usual Lorentz factor. Inserting the field (A3) in Eq. (A2) leads to

$$
\begin{aligned}
E_{\perp}(\omega) & =-\frac{\partial}{\partial b} \sqrt{\frac{2}{\pi}} \int_{0}^{\infty} d t \cos (\omega t) \frac{\gamma q}{R} e^{-R / a} \\
& =-\sqrt{\frac{2}{\pi}} \frac{q}{v} \frac{\partial}{\partial b} K_{0}\left(b \sqrt{(\omega / \gamma v)^{2}+1 / a^{2}}\right) ;
\end{aligned}
$$

see Ref. [17] for the last step. Using the relation $K_{0}^{\prime}=-K_{1}$ [18], we may express $E_{\perp}(\omega)$ in terms of the modified Bessel function $K_{1}$ as

$$
E_{\perp}(\omega)=\sqrt{\frac{2}{\pi}} \frac{q}{v b} x K_{1}(x)
$$


where $x$ is defined as

$$
x=\sqrt{(\omega b / \gamma v)^{2}+(b / a)^{2}} .
$$

Combining Eqs. (A1) and (A5) and substituting $c$ for $v$ finally gives

$$
\frac{d I}{d \omega d^{2} b}=\frac{q^{2}}{\pi^{2} c b^{2}}\left[x K_{1}(x)\right]^{2} .
$$

The results (A5) and (A7) are identical to those obtained for a point charge in the limit $\gamma \gg 1$ except that in the Coulomb case $x=\omega b / \gamma v$ [corresponding to $a \rightarrow \infty$ in Eq. (A6)]. Integration over impact parameters beyond a minimum value $d$ may be performed by the help of [17] and the recursion formulas for the modified Bessel functions [17,18] to yield Eqs. (8) and (9).
[1] S. P. Ahlen, Rev. Mod. Phys. 52, 121 (1980).

[2] B. A. Weaver and A. J. Westphal, Nucl. Instrum. Methods B 187, 285 (2002).

[3] A. H. Sørensen, AIP Conf. Proc. 680, 102 (2003).

[4] A. H. Sørensen, Nucl. Instrum. Methods B 230, 12 (2005). The full citation for Ref. [2] in this article is A. Belkacem and A. H. Sørensen, Radiat. Phys. Chem. 75, 656 (2006).

[5] C. A. Bertulani and G. Baur, Nucl. Phys. A458, 725 (1986).

[6] J. D. Jackson, Classical Electrodynamics (Wiley, New York, 1975).

[7] W. Heitler, The Quantum Theory of Radiation (Oxford University Press, London, England, 1954; reprint by Dover, New York, 1984).

[8] K. P. Schelhaas, J. M. Henneberg, M. Sanzone-Arenhövel, N. Wieloch-Laufenberg, U. Zurmühl, B. Ziegler, M. Schumacher, and F. Wolf, Nucl. Phys. A489, 189 (1988).

[9] J. C. Baggesen and A. H. Sørensen, Nucl. Instrum. Methods B 267, 2662 (2009).
[10] A. J. Baltz, G. Baur, D. d'Enterria, L. Frankfurt, F. Gelis, V. Guzey, K. Hencken, Yu. Kharlov, M. Klasen, S. R. Klein, V. Nikulin, J. Nystrand, I. A. Pshenichnov, S. Sadovsky, E. Scapparone, J. Seger, M. Strikman, M. Tverskoy, R. Vogt, S. N. White, U. A. Wiedemann, P. Yepes, and M. Zhalov, Phys. Rep. 458, 1 (2008); see chapter 7.

[11] J. Lindhard and A. H. Sørensen, Phys. Rev. A 53, 2443 (1996).

[12] S. Jeon, J. Kapusta, A. Chikanian, and J. Sandweiss, Phys. Rev. C 58, 1666 (1998).

[13] S. M. H. Wong, M. Belkacem, J. I. Kapusta, S. A. Bass, M. Bleicher, and H. Stöcker, Phys. Rev. C 63, 014903 (2000).

[14] V. Z. Jankus, Phys. Rev. 90, 4 (1953).

[15] G. Racah, Nuovo Cimento 14, 93 (1937).

[16] K. Hencken, D. Trautmann, and G. Baur, Phys. Rev. C 60, 034901 (1999).

[17] I. S. Gradshteyn and I. M. Ryzhik, Table of Integrals, Series, and Products (Academic Press, San Diego, 1980).

[18] Handbook of Mathematical Functions, edited by M. Abramowitz and I. A. Stegun (Dover, New York, 1972). 